\title{
Una nuova edizione democritea
}

Walter Leszl, I primi atomisti, Firenze: Olschki, 2009, pp. LVIII-450, con CD-ROM.

\section{GUGLIELMO RUIU / Roma /}

In un clima di rinnovamento degli studi sull'atomismo antico, Walter Leszl ci propone una nuova edizione dei testi riguardanti Leucippo e Democrito. A sua volta il supporto informatico propone, nell'ordine:

1. un Indice tematico,

2. un Indice dei principali termini, concetti e argomenti,

3. una Tavola di concordanza con le edizioni Diels-Kranz, Luria e Natorp (in quest'ultimo caso, limitatamente all'etica),

4. una Presentazione dei testi con esposizione dei criteri di raccolta del materiale e approfondimenti di carattere filologico,

5. un Ragguaglio sui testi qui introdotti per la prima volta e su quelli accantonati,

6. un Indice dei passi,

7. una Bibliografia (selettiva),

8. un Glossario dei termini greci e latini con traduzione italiana,

9. una serie di Supplementi all'Introduzione (vi si approfondiscono alcuni snodi fondamentali della tradizione democritea).

L'opera ha avuto una lunga gestazione, anche perché sono annunciati pure un commento più dettagliato, integrato con un'interpretazione complessiva del primo atomismo, una raccolta di testi greco-latini che ha funzionato da base per la presente edizione e una Introduzione agli atomisti che prenderà in considerazione anche gli atomisti minori. Nel frattempo viene offerta la raccolta dei testi in traduzione italiana, 
con introduzione e commento, una numerazione progressiva (simile a quella offerta dal Luria) e un'accurata suddivisione delle fonti in capitoli e paragrafi tematici.

Un'edizione dei testi democritei - e degli atomisti in generale - pone molte difficoltà, la maggiore delle quali è forse il fatto che, tolti i frammenti etico-politici, la netta maggioranza delle testimonianze sulla fisica - nel senso greco del termine - risale ad avversari dell'atomismo, sicché è difficile in molti casi separare, per così dire, la farina dalla crusca, cioè le citazioni, più o meno letterali, dal contesto critico o polemico in cui esse compaiono. Premesso che nessuna forma di classificazione è pacifica, e che fatalmente porta con sé vantaggi e svantaggi, occorre dire che l'ordinamento per contenuti - che è poi lo stesso utilizzato dai dossografi antichi - da un lato presenta l'indubbio vantaggio di organizzare il materiale in modo logico e uniforme (anche se le troppe suddivisioni hanno l'effetto di parcellizzare, verrebbe da dire di atomizzare, un pensiero originariamente complesso e articolato) nonché rendere più semplice il reperimento di informazioni dettagliate da parte del lettore, dall'altro però finisce per non evidenziare a sufficienza la distinzione fra testimonianza indiretta e frammento diretto, come pure le differenze di valore tra le fonti. Rileviamo anche l'arbitrarietà dei titoli o lemmi, la difficoltà di riportare a un'unica casella tematica testi che affrontano più temi, la rottura della continuità testuale. Per esempio non è facile, se non impossibile, separare nettamente, come fa Leszl, le sezioni dedicate alla sensazione rispetto a quelle sull'anima (significativamente il Luria aveva scelto di riportare il medesimo brano sotto distinti titoli, accorgimento respinto da Leszl). Del resto anche una terza via possibile, ossia l'ordinamento di tipo cronologico, può apparire ed è un metodo più neutro e oggettivo, ma di per sé non dice nulla sulla significatività o attendibilità delle fonti, né sui rapporti gerarchici tra di esse.

Se consideriamo lo stato della documentazione dossografica (che nel caso democriteo, si sa, coinvolge esclusivamente la teoria fisica), è innegabile che la distinzione tra testimonianza e frammento non è sempre agevole e in certi casi persino di scarsa utilità (per esempio nei passi brevissimi o incompleti) e che la differenza che corre fra riportare una testimonianza contenente una citazione e riportare un frammento inserito in un contesto può sembrare trascurabile (si vedano in proposito le incertezze di Diels-Kranz che talvolta classificano uno stesso passo come testimonianza e come frammento: è il caso, tra gli altri, del brano di Simplicio riportato come 68 A 67 e B 167 oppure di Stobeo che compare come 68 A 167 e B 170-171), ma è indubbio che il discorso non può valere per i frammenti etici, trattandosi di sentenze più o meno brevi senza contesto.

Consapevole di tutte queste difficoltà, Leszl finisce per adottare una soluzione che, benché un po' troppo macchinosa, potrebbe rappresentare un nobile compromesso, ossia affiancare ai testi una sigla letterale per indicarne la natura: $\mathrm{T}$ (pura testimonian$\mathrm{za}$ ), F (puro frammento), TF (testimonianza includente frammento), TC (testimonianza includente breve citazione) e così via. Lo stesso studioso mostra in verità qualche comprensibile incertezza nell'utilizzo di tante e tali formule, proponendo qua e là doppie sigle o rinunciando addirittura a offrirne (è quanto avviene con i passi degli Epicurei, difficilmente etichettabili). Un merito di questa edizione consiste nell'avere ampliato sensibilmente la documentazione preesistente, introducendo materiale o precedentemen- 
te scartato o passato inosservato o scoperto recentemente (è il caso di alcune testimonianze di Diogene di Enoanda), o ancora testi che, pur non riportando espressamente il nome di Leucippo e Democrito, contengono evidenti allusioni a questi ultimi: parliamo dei brani, soprattutto aristotelici, in cui si parla di "fisiologi" o "naturalisti". Si tratta in totale di una settantina di testi nuovi rispetto all'edizione Luria, che a sua volta raddoppiava di fatto la documentazione allestita da Diels-Kranz (a onor del vero va ricordato che lo stesso Diels nella quarta edizione dei Vorsokratiker auspicava per il capitolo su Democrito «eine übersichtlichere Form»). Le novità sono rappresentate soprattutto dai commentatori di Aristotele (Filopono in testa), ma anche da Plutarco e dallo stesso Aristotele.

Occorre contemporaneamente ammettere che in questo come in altri casi (es. la recente ediz. Wöhrle dei maestri di Mileto) l'arricchimento quantitativo delle fonti non è proporzionale alla significatività quantunque una parte del materiale disponibile sia stata scartata per varie ragioni. Tutto questo è ovviamente normale in un'edizione che vuole essere ampia ma selettiva, a patto che i criteri di esclusione e inclusione siano applicati con trasparenza e coerenza. A riguardo alcune scelte operate da Leszl lasciano qualche dubbio, come quella di escludere citazioni democritee spettanti a uno o più commentatori aristotelici quando si rilevano delle inesattezze. Ma l'imprecisione dell'informazione riportata da una fonte non sempre è un buon criterio di esclusione. Per fare un caso, la testimonianza di Olimpiodoro, a commento dei Metereologica di Aristotele, là dove assegna all'Abderita la teoria dei mondi infiniti, appare fortemente significativa e tutt'altro che trascurabile e scartabile, anche perché in linea con altre consimili testimonianze tradizionalmente registrate e apprezzate. Arbitraria sembra pure la scelta di includere solo una parte delle sentenze attribuite a Democrito nei florilegi bizantini, in base al criterio della presenza di una stessa sentenza in più fonti o al lume della «personale sensazione» di autenticità. La larga diffusione di un testo, infatti, non sarebbe, di per sé, prova della sua autenticità. È positivo, invece, che l'edizione Leszl spesso proponga brani più ampi che non nelle edizioni poziori, brani che in molti casi sono affiancati da una sintesi del contesto antecedente o seguente allo scopo di migliorare la comprensione e l'inquadramento dei singoli passi.

Sul piano del contributo critico, tra i vari meriti dell'autore c'è, oltre a una corretta e onesta rivalutazione dell'etica democritea, l'avere modificato, precisandola, la ricostruzione del Diels - in effetti troppo schematica - circa la tradizione dossografica relativa alla fisica, così com'è descritta nei Doxografi Greci (peraltro già sottoposta a energica revisione da Mansfeld e Runia): la linea, cioè, che parte dalle Opinioni dei fisici di Teofrasto e arriverebbe all'Antologia di Stobeo, precisamente ai primi due libri, passando attraverso i cosiddetti Vetusta Placita prima e i Placita di Aezio poi (questi ultimi, si sa, noti grazie all'epitome corrente col nome Placita philosophorum dello Pseudo-Plutarco). Leszl mostra bene per esempio come Simplicio, nel caso specifico di Democrito, attinga dichiaratamente al De Democrito di Aristotele e alla Fisica di Eudemo di Rodi piuttosto che alla ricordata opera teofrastea. Quanto all'ipotesi dell'esistenza di un'opera dossografica dovuta a Aezio come fonte - diretta o indiretta - della maggior parte del materiale dossografico successivo, lo studioso lascia prudentemente aperta la questione, dimostran- 
do comunque che certe opere non rientrano in tale schema: il riferimento va alla Historia philosopha pseudogalenica e alla Praeparatio evangelica di Eusebio di Cesarea, ambedue dipendenti direttamente non da Aezio ma dai Placita dello Pseudo-Plutarco.

Passiamo ora a rilevare alcuni punti deboli. Tralasciando rilievi di carattere editoriale (uso eccessivo delle parentesi integrative nel corpo del testo, indici non alfabetici ecc.), un limite evidente è il fatto che il testo proposto non sia innovativo rispetto alle edizioni già esistenti, mancando una nuova collazione dei manoscritti. A sua volta, l'apparato di note trascura molte questioni, anche capitali, di costituzione del testo (ad esempio il problema linguistico) e finisce spessissimo per omettere la varia lectio, anche in presenza di varianti e congetture fortemente significative. Il commento filosofico risulta quanto meno lacunoso e la letteratura critica sui singoli frammenti o testimonianze viene nel complesso ignorata.

Questa nuova edizione in ogni caso dà un nuovo e decisivo impulso agli studi democritei e si pone come strumento irrinunciabile per successive esplorazioni dell'atomismo antico. 